\title{
Aplicability of the Rao Mapping Function
}

\begin{tabular}{|l|l|l|}
\hline N.E. & Newton E. & Morton \\
\hline Newton E. Morton, Human Genetics Group, Level G, Princess Anne Hospital, Southampton S09 4HA (UK)
\end{tabular}

Seventy-five years ago Haldane [1] introduced a mapping function $\omega=f(\theta)$, where $\omega$ is a map distance as the mean number of exchanges per chromatid between two loci with recombination $\theta$. Assuming no interference, he obtained $\omega=[-\ln (1-2 \theta)] / 2$. He went on to show that triads of loci in Drosophila were much better described by a compound function $\omega$ $=0.7 \Theta+0.3[-\ln (1-2 \theta)] / 2$. This led to the mapping functions of Kosambi [2] and Carter and Falconer [3] which allow for progressively greater interference. None of these approaches was based on a biological model of 4-strand recombination or generalised to multiple loci: their justification was simply that they fit multiple pairwise data better than the model of no interference.

Seventeen years ago, Rao et al. [4] proposed a generalisation of these mapping functions that included all of them as special cases of a mapping parameter $p$, which they applied to multiple pairwise chiasmata in human spermatogen-esis [5]. They showed that $p$ for metacentric chromosomes (0.35) is intermediate between the Carter-Falconer (0.25) and Kosambi (0.5) values, but shows more interference $(0.11)$ for acrocentrics. They noted that $\omega>\theta$ for the classical mapping functions, but that values for $\omega$ for some small values of $p$ 'are negligibly smaller than the corresponding $\theta$, since equation 1 does not impose $\omega>\theta$ for small values of $p$. It is a matter of taste, with no practical consequence, whether one accepts the least squares estimate of $p$ or imposes $\omega>\theta$ ". This point has recently been reiterated without attribution in this journal [6]. The differences under discussion are in the third decimal place, much smaller than the effect of typing errors and not significant even in large samples. It would take a million error-free informative meioses to reach significance in the example given.

Lalouel [7] showed that multiple pairwise data for chromosome 3 of Drosophila are consistent with $p=0.45$. Subsequent studies confirmed that pericentric loci have interference at about the Kosambi level, but interference is greater within a chromosome arm [8]. The hypothesis of no interference is rejected in favour of the Rao mapping function at a very high significance level [9-11]. Interference is partly confounded with classification errors but the two effects are separable [12]. There is good agreement between map lengths from chiasmata in males and from multiple pairwise linkage data fitted by the Rao mapping function. There does not seem to be a sex difference in interference despite the large difference in recombination.

All mapping functions are approximate, but this does not make them invalid. The Rao function has demonstrated its ability in multiple pairwise analysis to give significantly higher likelihoods than specified alternatives. It is now commonplace to assemble lods from many samples and then analyse several hundred loci in sex-specific linkage maps with 
simultaneous estimation of the mapping parameter and error frequency. In contrast, it is a major accomplishment to map half a dozen loci in a single sample by multipoint methods without error filtration. The prevailing standard is to neglect error and interference in calculating multipoint likelihoods [13], which in the presence of typing error give more mistakes in order than multiple pairwise methods [14],

The letter of Weeks [6] will have served a purpose if it leads to more general understanding of the objectives and accomplishments of mapping functions in multiple pairwise analysis and of the limitations of multipoint mapping.

0453-017858.00/0

\section{References}

Haldane JBS: The combination of linkage values and the calculation of distance between the loci of linked factors. J Genet 1919;8:299-309. Kosambi DD: The estimation of map distance from recombination values. Ann Eugen 1944; 12:172-175.

Carter TC, Falconer DS: Stocks for detecting linkage in the mouse and the theory of their design. J Genet 1951;50:307-323.

Rao DC, Morton NE, Lindsten J, Hulten M, Yee S: A mapping function for man. Hum. Hered. 1977;27: 94-104.

Hulten M: Chiasma distribution at diakinesis in the normal human male. Hereditas 1974;76:55-78.

Weeks DE: Invalidity of the Rao map function for three loci. Hum. Hered. 1994;44:178-180. Lalouel JM: Linkage mapping from pair-wise recombination data. Heredity 1977;38:61-77. Morton NE, MacLean CJ, Lew R: Tests of hypotheses on recombination frequencies. Genet Res 1985; 45:279-286.

Morton NE, Collins A: Standard maps of chromosome 10. Ann Hum Genet 1990;54:235251.

10 Collins A, Keats BJ, Dracopoli N, Shields DC, Morton NE: Integration of gene maps: Chromosome 1. Proc Natl Acad Sci USA 1992;89:4598-4602.

Wang LH, Collins A, Lawrence S, Keats BJ, Morton NE: Integration of gene maps: Chromosome X. Ge-nomics 1994;22:590-604.

Shields DC, Collins A, Buetow KH, Morton NE: Error filtration, interference, and the human linkage map. Proc Natl Acad Sci USA 1991 88:6501-6505.

Weeks DE, Lathrop GM, Ott J Multipoint mapping under genetic interference. Hum. Hered. 1993;43 86-97.

Buetow KH: Influence of aberrant observations on high-resolution linkage analysis outcomes. Am J Hum Genet 1991;49:985-994.

Daniel E. Weeks

Reply

A mathematical model of a biological phenomenon is presumably better if it reflects our knowledge of the phenomenon as accurately as possible. In terms of genetic mapping with multipoint likelihoods, a mathematical model that takes interference into account should lead to more accurate results than one that ignores interference. Weeks et al. [1, 2] have developed and applied programs that permit mapping under different models of interference but, as Morton eloquently points out, these methods are computationally very demanding. 
Thus, due to computational constraints, one may wish to employ the multiple pairwise methods, which permit the simultaneous inclusion of both error and interference [3]. One way of modeling the effects of interference is to use map func-

Received: July 30, 1994 Accepted: August 8,1994

Daniel E. Weeks, PhD University of Pittsburgh Department of Human Genetics 130 DeSoto Street, A300 C¹/8abtree Hall Pittsburgh, PA 15261 (USA)

179

tions, which define the relationship between nonadditive recombination fractions $(\theta)$ and additive genetic distances $(\omega)$. I previously pointed out that the Rao map function [4] is 'invalid' according to the following definition:

A map function $\mathrm{M}(\omega)$ is valid for three loci if for all values of $\theta \mathrm{i}$ and $\theta 2$ between 0.00 and $0.50, \theta 3=\mathrm{M}[\mathrm{M}-'(\theta \mathrm{i})+\mathrm{M}-1(1 / 8)]$ satisfies $\theta \downarrow+$

$02 \geq 03$.

Morton claims that the invalidity of the Rao map function had already been pointed out by Rao et al. [5] in their original paper, since they stated that the Rao map function generates $\omega$ $<\theta$ for small values of $p$, where $p$ is the Rao map function parameter which is an indicator of the strength of interfer-

ence. However, generating a $\omega<\theta$ is not logically the same as being invalid. For example, consider the (modified Morgan) map function $\mathrm{M}(\omega)=1.1 \omega=\theta$. This has the inverse function $\omega=\mathrm{M}-{ }^{\prime}(\theta)=0.9 \Theta$, so we have $\omega<\theta$ always. However, $\theta 3=\mathrm{M}[0.9 \theta !+0.9 \Theta 2]=(1.1)(0.9)(\theta \mathrm{l}$ $+\theta 2)=0.99(\theta \mathbf{l}+\theta 2)$, which implies that $\theta 3<\theta$ ! $+\theta 2$. While this map function does not satisfy the constraint that $\theta$ should always be less than 0.50 , for small values of $\theta$ it is valid, yet always generates $\omega<\theta$. Thus, I hope my previous letter [4] may be viewed as contributing to a deeper understanding of the Rao map function, rather than as a reiteration 'without attribution' of a previously made point.

References

Weeks DE, Lathrop GM, Ott J: Multipoint mapping under genetic interference. Hum. Hered. 1993;43: 86-97.

Weeks DE, Ott J, Lathrop GM: Detection of genetic interference: Simulation studies and mouse data. Genetics 1994;136:1217-1226. Shields DC, Collins A, Buetow KH, Morton NE: Error filtration, interference, and the human linkage map. Proc Natl Acad Sci USA 1991; 88:6501-6505.

Weeks DE: Invalidity of the Rao map function for three loci. Hum. Hered. 1994;44:178-180. Rao DC, Morton NE, Lindsten J, Hulten M, Yee S: A mapping function for man. Hum. Hered. 1977;27: 99-104.

Newton E. Morton

Reply

At the risk of introducing some biology into a discussion that is drifting, map distance in morgans $\omega$ is the mean number of exchanges per strand. The Weeks counterexample violates this definition. Could we return to the real world where $\theta>\omega$ is not a mapping function? 
Received: August 1,1994 Accepted: August 10,1994 